\title{
Uso de lodo de esgoto na produção de tapetes de grama esmeralda
}

\author{
Use of sewage sludge in zoysiagrass production
}

\author{
Clarice Backes ${ }^{\mathrm{I}}$ Leonardo Theodoro Büll' ${ }^{\mathrm{II}}$ Leandro José Grava de Godoy ${ }^{\text {III }}$ \\ Roberto Lyra Villas Bôas ${ }^{\mathrm{II}}$ Claudinei Paulo de Lima ${ }^{\mathrm{IV}}$ Eder Carlos Pires $^{\mathrm{V}}$
}

RESUMO

Com objetivo de avaliar o efeito do lodo de esgoto na formação e qualidades de tapetes de grama, foi instalado, em Itapetininga, São Paulo (SP), o experimento utilizando grama Zoysia japonica Steud., conhecida como esmeralda. $O$ delineamento experimental utilizado foi o de blocos ao acaso em parcelas subdivididas, com quatro repetições, sendo as parcelas principais constituídas de cinco doses de lodo (0, 10, 20, 30 e 40Mg ha ${ }^{-1}$, base seca), mais um tratamento com NPK, de acordo com as necessidades da cultura e as subparcelas com dois sistemas de manejo (com e sem o uso de escarificador utilizado para romper uma camada superficial compactada). Utilizou-se o lodo da Estação de Tratamento de Esgoto de Jundiaí, SP. As doses de lodo aplicadas correspondem às doses de 100, 200, 300 e 400 $\mathrm{kg} \mathrm{ha}^{-1}$ de nitrogênio. Após 165 dias da aplicação do lodo de esgoto, a dose de $31 \mathrm{Mg} \mathrm{ha}^{-1}$ permitiu o fechamento completo (100\% da taxa de cobertura do solo) $e$ a maior resistência dos tapetes de grama. O mesmo resultado foi encontrado quando utilizou-se a adubação química. A testemunha e as parcelas que receberam $10 \mathrm{Mg} \mathrm{ha}^{-1}$ de lodo não formaram tapete. O lodo de esgoto aplicado em superfície promoveu redução da massa dos tapetes de grama esmeralda. O uso do escarificador reduziu a resistência dos tapetes de grama esmeralda. Os valores de metais pesados encontrados no lodo de esgoto estavam abaixo do limite estabelecido pelas normas que regulamenta a utilização do lodo de esgoto na agricultura.

Palavras-chave: Zoysia japonica Steud., nitrogênio, cobertura do solo, sistemas de manejo

\begin{abstract}
Aiming to evaluate sewage sludge effect in the grass sod qualitiy and formation it was installed in Itapetininha - SP one experiment using Zoysia japonica Steud. The experimental design adopted was randomized blocks with split plot design, with four replications, the main plots consisting of five doses of sludge $\left(0,10,20,30\right.$ and $40 \mathrm{Mg} \mathrm{ha}^{-1}$, a dry basis), another treatment with NPK, according to the requirement of the culture, and subplots with two management systems (with and without chisel use, to break a compacted surface layer). The sludge used came from "Estação de Tratamento de Esgoto de Jundiaí/SP". The doses of sludge applied correspond to the doses of 100, 200, 300 and $400 \mathrm{~kg} \mathrm{ha}^{-1}$ of nitrogen. 165 days after sewage sludge application, the dose of $31 \mathrm{Mg} \mathrm{h}^{-1}$ allowed the complete closing (100\% of soil cover rate) and greater resistance of the zoysiagrass sod. The same results were found when chemical fertilization was used. The control and the plots that received $10 \mathrm{Mg} \mathrm{ha} \mathrm{g}^{-1}$ of sludge did not formed sod. The sewage sludge applied to surface promoted reduction of zoysiagrass sod mass. The use of surface chisel reduced the strength of zoysiagrass sod. The heavy metals values found in sewage sludge were below the limit set by the rules governing the sewage sludge use in agriculture.
\end{abstract}

Key words: Zoysia japonica Steud., nitrogen, soil cover, systems of handling.

\section{INTRODUÇÃO}

Uma das principais gramas comercializadas no Brasil é a Zoysia japonica Steud., conhecida como

\footnotetext{
IDepartamento de Produção Vegetal/Horticultura, Faculdade de Ciências Agronômicas (FCA), UNESP, CP 237, 18610-907, Botucatu, SP, Brasil. E-mail: claricebackes@hotmail.com. Autor para correspondência.

"Departamento de Recursos Naturais, Ciência do Solo, FCA, UNESP, Botucatu, SP, Brasil.

IIIUnidade Diferenciada de Registro, UNESP, Registro, SP, Brasil.

${ }^{\text {IV}}$ Departamento de Produção Vegetal/Agricultura, FCA, UNESP, Botucatu, SP, Brasil.

${ }^{\mathrm{VI}}$ Curso de Agronomia, FCA, UNESP, Botucatu, SP, Brasil.
} 
esmeralda, que apresenta crescimento rizomatosoestolonífero, com folhas de textura fina a média, com excelente densidade e coloração verde-médio (GURGEL, 2003).

Como as áreas de produção de gramas são muito compactadas, sendo utilizadas por vários anos após sua implantação sem que se mobilize o solo de modo efetivo, a quantidade de fertilizantes adicionada nesse sistema deve ser alta para elevar a concentração de nutrientes no solo e suprir a redução de aeração nas raízes e a absorção ativa de nutrientes, já que a compactação acaba diminuindo a eficiência de absorção de nutrientes pelas plantas (GODOY \& VILLAS BÔAS, 2003). Como prática no sistema de produção de grama, os produtores utilizam um escarificador superficial do solo, a fim de romper a camada superficial compactada, melhorando assim a eficiência da adubação, que é realizada superficialmente.

O lodo de esgoto apresenta disposição final problemática e frequentemente negligenciada, comprometendo parcialmente os efeitos benéficos da coleta e do tratamento de esgoto. A utilização agrícola desse resíduo já ocorre atualmente em muitos países. O interesse pelo lodo de esgoto como fertilizante não decorre apenas da presença de nutrientes em sua composição, principalmente nitrogênio, fósforo e micronutrientes, mas também do seu teor de matéria orgânica (SANEPAR, 1997).

O nitrogênio é o nutriente requerido em maiores quantidades pelas gramas (BOWMAN etal., 2002)e também está presente em maior concentração no lodo de esgoto.

Diversos trabalhos têm mostrado aumentos na produção de matéria seca e grãos por espécies de interesse agronômico cultivadas em solos tratados com lodo de esgoto (DA ROS et al., 1993; BERTON et al., 1997). Em alguns casos, os aumentos são equiparáveis ou superiores aos obtidos com a adubação mineral recomendada para a cultura (DAROS et al., 1993; SILVA et al., 2001). Porém, em nível nacional, não há informação da aplicação de lodo na formação de tapetes de grama. MURRAY (1991), avaliando a aplicação de lodo de esgoto para a produção de grama azul de Kentucky (Poa pratensis L.) e bermuda (Festuca rubra L.), nos Estados Unidos, verificou que doses crescentes do lodo até $200 \mathrm{Mg} \mathrm{ha}^{-1}$, incorporadas ou aplicadas em superfície, causaram aumento do $\mathrm{pH}$, maior capacidade de troca catiônica, maior agregação, maior teor de matéria orgânica e maior retenção de água do solo. Em contraste, a densidade do solo e o peso dos tapetes por unidade de área diminuíram, o que é uma vantagem, pois pode permitir o transporte de maior número de tapetes sem ultrapassar o limite de peso da carga. Dessa forma, o lodo de esgoto poderia ser uma alternativa de fonte de nutrientes para diminuir os custos de produção e transporte, aumentando assim os lucros dos produtores, pois somente a adubação da grama representa $21,5 \%$ dos custos de produção (AGRIANUAL, 2006).

A presença de metais pesados constitui uma limitação no uso do lodo na agricultura. Esses metais podem expressar seu potencial poluente diretamente nos organismos do solo, pela disponibilidade às plantas em níveis fitotóxicos, além da possibilidade de transferência para a cadeia alimentar por meio das próprias plantas ou pela contaminação das águas de superfície e subsuperfície. Porém, alguns estudos realizados mostram que os níveis de metais pesados encontrados nesse material mostram-se abaixo dos níveis críticos restritivos para sua utilização agrícola, o que permite sua aplicação ao solo dentro de limitações toleráveis de impacto ambiental (SILVA, et al., 2002).

Assim, objetivou-se com o presente trabalho avaliar o uso do lodo de esgoto com e sem escarificação superficial do solo na produção e qualidade de tapetes de grama esmeralda.

\section{MATERIAL E MÉTODOS}

O experimento foi instalado e conduzido em propriedade de grama no Município de Itapetininga São Paulo (SP), localizada nas coordenadas geográficas $23^{\circ}{ }^{\circ} 1^{\text {' }}$ de latitude sul e 4803’ de longitude oeste de Greenwich e altitude média de $636 \mathrm{~m}$. A área vinha sendo utilizada para a produção comercial de grama, colhida mecanicamente em tapetes, há cerca de 10 anos. O solo é classificado como Latossolo Vermelho Distrófico, textura argilosa, conforme nomenclatura do Sistema Brasileiro de Classificação de Solos (EMBRAPA, 1999). Os dados de precipitação indicaram 277mm de chuva entre os meses de outubro de 2005 e maio de 2006, sendo notado déficit hídrico nos meses de novembro, janeiro e maio avaliado por meio da relação precipitação/ evapotranspiração. A temperatura média ficou em $23^{\circ} \mathrm{C}$, sendo a mínima obtida em maio de $18^{\circ} \mathrm{C}$, e a máxima obtida nos meses de janeiro e março de 26 e $25^{\circ} \mathrm{C}$.

De acordo com o resultado da análise o solo possuía as seguintes características químicas, antes da instalação do experimento: $\mathrm{pH}\left(\mathrm{CaCl}_{2}\right)$ de 4,6; $38 \mathrm{~g}$ $\mathrm{dm}^{-3}$ de M.O.; $3 \mathrm{mg} \mathrm{dm}^{-3} \mathrm{de}$ P (resina); 54; 0,9; 26 e 7mmol $\mathrm{dm}^{-3}$ de $\mathrm{H}^{+}+\mathrm{Al}^{+3}$, K, Ca e $\mathrm{Mg}$, respectivamente; saturação por bases (V) de 36\%. A composição granulométrica do solo foi de 90,671 e $239 \mathrm{~g} \mathrm{~kg}^{-1}$ de areia, argila e silte, respectivamente.

Foi utilizada a espécie Zoysia japonica Steud., conhecida como grama esmeralda, que tem 
hábito de crescimento rizomatoso e, portanto, pode ser colhida em área total, visto que, após a colheita, ficam rizomas subsuperficiais capazes de brotarem e cobrir novamente o solo.

O delineamento experimental utilizado foi o de blocos ao acaso em parcelas subdivididas, com quatro repetições. As parcelas principais foram constituídas de cinco doses de lodo $(0,10,20,30$ e $40 \mathrm{Mg}$ ha $^{-1}$, base seca), mais um tratamento com fertilizante misto NPK, de acordo com as necessidades da cultura, e as subparcelas de dois sistemas de manejo (com e sem o uso de escarificador superficial). O escarificador superficial denominado "estrelinha" consiste de grade de discos recortados em forma de estrela, contendo 10 discos distanciados entre si em 10 $\mathrm{cm}$, que acoplada a um trator é utilizada para romper uma camada superficial compactada. Foram realizadas quatro passagens do escarificador nas parcelas que receberiam esse tratamento, a uma profundidade de, aproximadamente, $2,0 \mathrm{~cm}$, em um único sentido. As parcelas experimentais foram de 2,2 x 5 metros, e a bordadura foi de $0,5 \mathrm{~m}$ em cada extremidade.

O lodo de esgoto utilizado foi proveniente da estação de tratamento de esgoto da cidade de Jundiaí, SP, apresentando a concentração de 32; 18; 2,1; 13,1; 2,8; 24; 520 e 289g kg ${ }^{-1}$ de N, P, K, Ca, Mg, S, M.O. e carbono orgânico, respectivamente; 722; 28.800; 674; 500; 0,59; 7,21; 152,7; <0,1; 34,5 e 184,4mg kg-1 de Cu, Fe, Mn, Zn, As, Cd, Cr, Hg, Ni e Pb, respectivamente, umidade de 68\%; relação C/N 9; e pH 5,9. As doses de lodo aplicadas correspondem a 100, 200, 300 e 400 kg ha ${ }^{-1}$ de nitrogênio disponível, calculadas com base no teor de $\mathrm{N}$ presente no lodo, na umidade do lodo, na taxa de mineralização de $30 \%$ ao ano e na necessidade da cultura.

A calagem foi realizada na área total, aplicando-se sobre a superfície do solo $1,2 \mathrm{Mg} \mathrm{ha}^{-1}$ de calcário dolomítico (PRNT de 91\%), considerando a saturação por bases desejada igual a $60 \%$. O lodo de esgoto foi aplicado em uma única vez 20 dias após a colheita dos tapetes e espalhado sobre a superfície do solo, imediatamente após a aplicação do calcário. Nas parcelas que receberam a adubação química, aplicaramse $500 \mathrm{~kg} \mathrm{ha}^{-1}$ do fertilizante misto de fórmula 04-14-08, 35 dias após a colheita dos tapetes, e o restante do nitrogênio do potássio foi parcelado em três vezes, sendo a dose total aplicada de $300 \mathrm{~kg} \mathrm{ha}^{-1} \mathrm{de} \mathrm{N}$. A fonte de $\mathrm{N}$ utilizada foi a uréia, aplicada manualmente, na superfície do solo aos 95 (17/12/06), 140 (01/02/07) e 180 (13/03/07) dias após o corte do tapete anterior, sendo essas parcelas irrigadas até 24 horas após a aplicação. Devido ao baixo teor de potássio presente no lodo de esgoto, foi aplicada a dose de $\mathrm{K}_{2} \mathrm{O}$ de $200 \mathrm{~kg}$ $\mathrm{ha}^{-1}$, forma de $\mathrm{KCl}$, parcelado em três vezes.
O corte dos tapetes foi realizado mecanicamente por meio de colhedora acoplada ao trator. No dia anterior, a área foi irrigada, e foi passado rolo compactador sobre a área experimental por três vezes até que se conseguisse um tapete com características de comercialização.

A taxa de cobertura do solo (TCS) pela grama foi avaliada por meio da análise de imagem digital, conforme metodologia descrita por GODOY (2005), aos 45, 75, 105, 135, 165 e 195 dias após a aplicação do lodo de esgoto (DAA).

Após o corte, foram determinadas a massa e a resistência de cada tapete ao rompimento, sendo determinado por meio de uma balança a massa necessária para rompê-lo. Para essa determinação, foram utilizados três tapetes para cada repetição.

Para a determinação de metais pesados, foram coletadas amostras de solo na camada de 0 a $20 \mathrm{~cm}$ com trado tipo sonda após a colheita dos tapetes. Utilizou-se como extrator o DTPA, e as determinações foram realizados por espectofotometria de emissão em plasma (ICP-AES).

Os resultados foram submetidos à análise estatística utilizando o software “SISVAR”, versão 4.2.

\section{RESULTADOS E DISCUSSÃO}

A TCS foi influenciada apenas pelas doses de lodo de esgoto. Para os sistemas de manejo, não houve diferença estatística. Na tabela 1 , estão representadas as equações de regressão da TCS em função das doses de lodo aos 45, 75, 105, 135, 165, 195 dias após a aplicação. Aos 45 dias após a aplicação do lodo, a dose de $38 \mathrm{Mg} \mathrm{ha}^{-1}$ possibilitou a TCS de 85,35\%. Aos 75 dias, houve aumento linear da TCS com o aumento das doses aplicadas, sem alcançar um ponto de máximo. Nas épocas de 105 e 135 DAA, a dose de $33 \mathrm{Mg} \mathrm{ha}^{-1}$ de lodo de esgoto promoveu taxas de cobertura do solo pela grama de 99,0 e 99,8\%, respectivamente. Aos 165 DAA, de acordo com a equação ajustada, a dose de lodo de $31 \mathrm{Mg} \mathrm{ha}^{-1}$ permitiu a cobertura total do solo pela grama esmeralda (TCS de 100\%). Segundo CHRISTIANS (1998), a TCS pode indicar a velocidade de produção do tapete, uma vez que a grama esmeralda, quando cobre totalmente a superfície do solo, também já produziu rizomas e raízes sob a superfície do solo, dando a estrutura para o tapete a ser cortado.

Com a TCS de 100\% aos 165 dias após a aplicação do lodo, verifica-se que o tempo de formação de tapetes de grama esmeralda foi menor em relação ao tempo médio de 12 meses para a colheita do tapete de grama esmeralda, no Brasil, citado por PIMENTA(2003). 
Tabela 1 - Equação de regressão para taxa de cobertura do solo pela grama $Z$. japonica (esmeralda), em função de doses de lodo de esgoto aos 45, 75, 105, 135, 165 e 195 dias após a aplicação (DAA).

\begin{tabular}{clc}
\hline & \multicolumn{1}{c}{ Equação de regressão } & $\mathrm{R}^{2}$ \\
\hline Dias após a aplicação (mês/ano) & $y=-0,0185^{* *} x^{2}+1,41^{* *} x+58,48$ & 0,99 \\
$75(\mathrm{dez} / 05)$ & $y=0,7114^{* *} x+67,246$ & 0,96 \\
$105(\mathrm{jan} / 06)$ & $y=-0,0164^{* *} x^{2}+1,092^{* *} x+80,905$ & 0,98 \\
$135(\mathrm{fev} / 06)$ & $y=-0,0122^{* *} x^{2}+0,8071^{* *} x+86,409$ & 0,99 \\
$165(\mathrm{mar} / 06)$ & $y=-0,0127^{* *} x^{2}+0,7947^{* *} x+87,829$ & 0,98 \\
$195(\mathrm{abr} / 06)$ & $y=-0,0116^{* *} x^{2}+0,7237^{* *} x+89,152$ & 0,99 \\
\hline
\end{tabular}

$\stackrel{* * *}{*}$ - significativo a 5 e $1 \%$.

KOSKE (1994) citou o período de 11 a 18 meses para a formação do tapete de Zoysia, no Estado de Lousiana, centro-sul dos Estados Unidos.

Verifica-se, na figura 1, que o maior aumento na taxa de cobertura do solo ocorreu aos 45 dias após a aplicação do lodo, aumentando cerca de $26 \%$ de cobertura do solo pela grama em comparação com as parcelas que não receberam o lodo. GODOY et al. (2007) ressaltam a importância da adubação de base em um primeiro arranque no crescimento da parte aérea, principalmente, em meses com temperatura adequada. Aos 105 dias após a aplicação do lodo, março de 2006, verificou-se que o solo estava praticamente coberto pela grama, apresentando 98 e $99 \%$ de cobertura com as doses de 30 e $40 \mathrm{Mg} \mathrm{ha}^{-1}$ de lodo, respectivamente. Com as elevadas temperaturas e precipitações ocorridas em janeiro (105DAA), possivelmente houve uma maior liberação de $\mathrm{N}$ do lodo, proporcionando assim um maior crescimento da grama. Segundo BOEIRA (2004), o lodo aplicado em campo pode liberar, à solução do solo, grande quantidade de $\mathrm{N}$ mineral nos primeiros dias após a aplicação, se houver condições propícias para a mineralização da matéria orgânica. As quantidades mineralizadas vão diminuindo ao longo do tempo, com tendência de estabilização por volta dos três meses, ou seja, a mineralização torna-se lenta, mas contínua.

A adubação química proporcionou baixas taxas de cobertura até os 95 dias após o corte do tapete anterior (Figura 1), sendo superior apenas à dose zero, porque haviam sido aplicados apenas $20 \mathrm{~kg}$ ha $^{-1}$ de N. A primeira adubação de cobertura, aos 95DAC, proporcionou aumento de 30\% na TCS, maior que o proporcionado pelas doses de lodo de esgoto. Fica evidente a disponibilidade mais rápida do nitrogênio proveniente do fertilizante mineral em relação ao lodo de esgoto. Nas demais épocas avaliadas, a adubação química proporcionou TCS semelhante às maiores doses de lodo aplicadas. As gramas que não receberam adubação nem lodo de esgoto e as que receberam $10 \mathrm{Mg} \mathrm{ha}^{-1}$ de lodo não formaram tapete, não chegando a cobrir o solo totalmente até o final do experimento (195DAA).

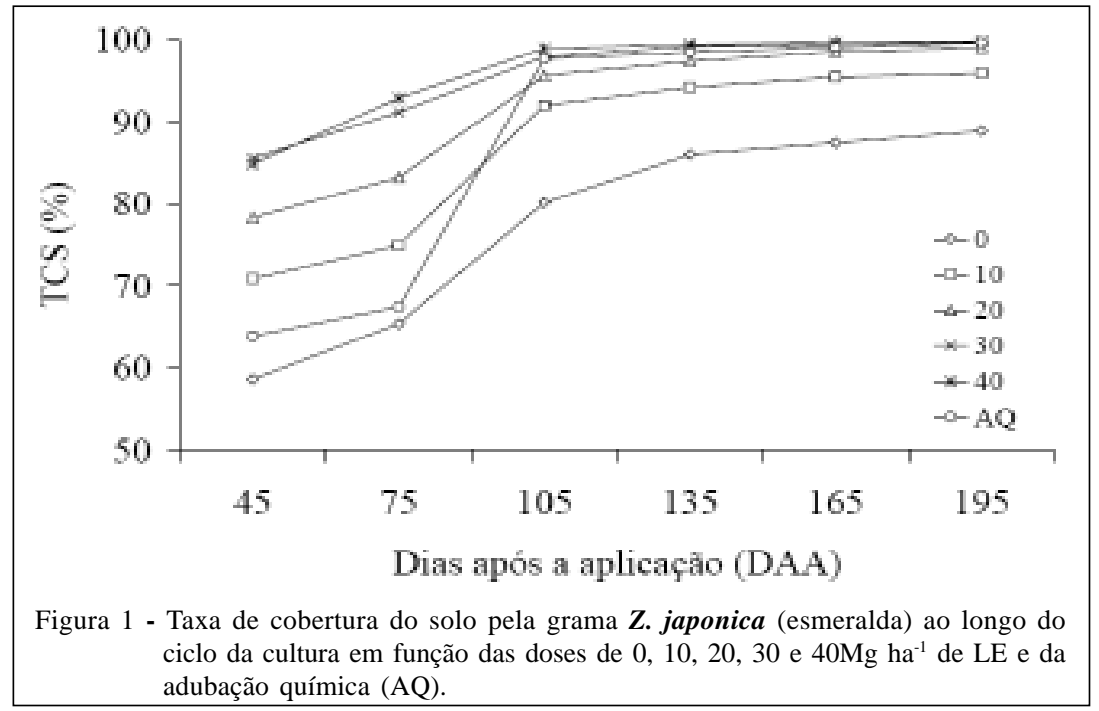

Ciência Rural, v.39, n.4, jul, 2009. 
Para a massa de tapetes, também houve diferença significativa apenas para as doses de lodo utilizadas, em que os sistemas de manejo não tiveram influência. Na figura 2a, observa-se que houve uma redução do peso dos tapetes com o aumento das doses de lodo de esgoto. Quando comparado com a adubação química, nota-se que, na dose de $40 \mathrm{Mg} \mathrm{ha}^{-1}$, houve redução de $13 \%$ do peso dos tapetes. Essa característica tem uma implicação prática muito importante, uma vez que, com a massa menor, consegue-se levar maior número de tapetes com a mesma carga. MURRAY (1991), avaliando a aplicação de 200 $\mathrm{Mg} \mathrm{ha}^{-1}$ de lodo de esgoto para a produção de grama azul de Kentucky (Poa pratensis L.) e bermuda (Festuca rubra L.), também verificou a diminuição do peso dos tapetes por unidade de área.

Para a resistência dos tapetes, houve interação entre as doses de lodo e os sistemas de manejo. Quando utilizou-se o escarificador superficial, a máxima resistência do tapete $(22,5 \mathrm{~kg})$ foi alcançada com a dose de $25 \mathrm{Mg} \mathrm{ha}^{-1}$ de lodo (Figura 2b). Sem o uso do escarificador, verifica-se que a máxima resistência do tapete foi encontrada com a dose de $31 \mathrm{Mg} \mathrm{ha}^{-1}$, e a adubação química apresentou resultados semelhantes. O uso da estrelinha não influenciou a velocidade de fechamento do tapete e ainda promoveu corte das raízes e rizomas, diminuindo, dessa forma, a resistência dos tapetes.

A dose de $40 \mathrm{Mg} \mathrm{ha}^{-1}$ promoveu uma rápida cobertura do solo (99\% aos 105DAA), porém houve redução na resistência dos tapetes de grama esmeralda produzidos com essa dose. Segundo KOSKE (1994), a utilização de doses muito elevadas de nitrogênio e a formação muito rápida do tapete podem prejudicar a liftability (capacidade de ser manuseado) pela redução no crescimento dos rizomas, principais responsáveis pela resistência do tapete (CHRISTIANS, 1998). Na produção de gramas em tapete, o crescimento de raízes e de rizomas é mais importante que o crescimento da parte aérea, pois terá mais influência para a formação do tapete resistente na colheita e para o manuseio, aumentando o rendimento da área. A maior resistência dos tapetes foi obtida com a dose de $31 \mathrm{Mg} \mathrm{ha}^{-1}$ de lodo, que é também a dose que promoveu a formação de tapetes de grama esmeralda em menor tempo.

Com referência aos teores de metais, observa-se que os valores encontrados no lodo de esgoto estão bem abaixo do limite estabelecido pelas normas do CONAMA (2006), que regulamenta a utilização do lodo de esgoto na agricultura. A concentração máxima permitida é de $1500 \mathrm{mg} \mathrm{kg}^{-1}$ para $\mathrm{Cu}, 2800 \mathrm{mg} \mathrm{kg}^{-1}$ para Zn, 41 $\mathrm{mg} \mathrm{kg}^{-1}$ para As, 39 $\mathrm{mg} \mathrm{kg}^{-1}$ para Cd, $1000 \mathrm{mg} \mathrm{kg}^{-1}$ para Cr, $17 \mathrm{mg} \mathrm{kg}^{-1}$ para Hg, 420mg $\mathrm{kg}^{-1}$ para Ni e $3000 \mathrm{mg} \mathrm{kg}^{-1}$ para $\mathrm{Pb}$. Com base na maior dose de lodo de esgoto aplicada no solo $\left(40 \mathrm{Mg} \mathrm{ha}^{-1}\right)$, foram adicionados aproximadamente 0,$023 ; 0,30 ; 29$; 6,11; 0,004; 1,38; 7,38 e 20kg ha ${ }^{-1}$ de As, Cd, Cu, Cr, Hg, $\mathrm{Ni}, \mathrm{Pb}$ e Zn, respectivamente.

A aplicação superficial do lodo não causou problemas de poluição ambiental, considerando a presença do teor disponível de metais pesados no solo após o corte dos tapetes de grama, nos quais os teores médios encontrados, para a camada de $0-20 \mathrm{~cm}$ de profundidade, quando aplicados $40 \mathrm{Mg} \mathrm{ha}^{-1}$ foram: $0,191 \mathrm{mg} \mathrm{dm}^{-3}$ de As; $0,149 \mathrm{mg} \mathrm{dm}^{-3}$ de Ni; 0,679mg dm ${ }^{-3}$ de $\mathrm{Pb}$; 5,55mg dm $\mathrm{m}^{-3}$ de $\mathrm{Cu} ; 3,4 \mathrm{mg} \mathrm{dm}^{-3}$ de $\mathrm{Zn}$. Esses valores não são muito diferentes de quando foi realizada a adubação química: 0,214; 0,134; 0,692; 4,65 e 0,5mg $\mathrm{dm}^{-3}$ de As, Ni, $\mathrm{Pb}$, Cu e Zn, respectivamente. Os metais $\mathrm{Cd}$, Cr e Hg apresentaram-se em concentrações abaixo do limite de determinação do método analítico empregado.

Além disso, praticamente todo o lodo aplicado é levado juntamente com o tapete, o que permitiria aplicação prolongada de lodo na mesma área, visto que a quantidade de metais que ficam no solo é muito baixa.
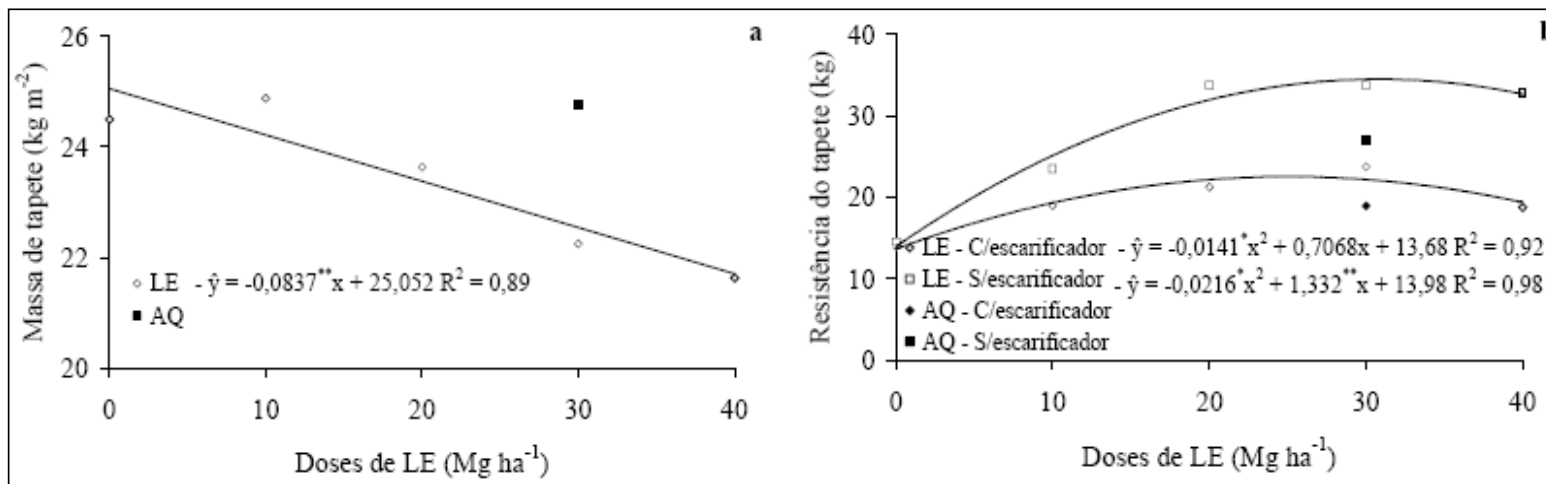

Figura 2 - Massa de tapete da grama Z. japonica (esmeralda), em função de doses de lodo de esgoto (LE) e da adubação química (AQ) (a); Resistência do tapete de grama $\mathbf{Z}$. japonica (esmeralda), em função de doses de lodo de esgoto (LE) e da adubação química (AQ) e dos sistemas de manejo (b). 


\section{CONCLUSÕES}

A dose de $31 \mathrm{Mg} \mathrm{ha}^{-1}$ de lodo de esgoto permitiu o fechamento completo do tapete de grama. Esse mesmo resultado foi encontrado quando utilizouse a adubação química. A testemunha e as parcelas que receberam $10 \mathrm{Mg} \mathrm{ha}^{-1}$ de lodo não formaram tapete.

Houve redução na massa dos tapetes de grama com o aumento das doses de lodo aplicadas e, com a utilização do escarificador, houve diminuição da resistência dos tapetes de grama.

Os valores de metais pesados presentes no lodo estavam abaixo do limite estabelecido pelas normas que regulamenta a utilização do lodo de esgoto na agricultura.

\section{AGRADECIMENTO}

À Fundação de Amparo à Pesquisa do Estado de São Paulo (FAPESP), pelo auxílio financeiro concedido.

\section{REFERÊNCIAS}

AgrianUAL: Anuário Estatístico da Agricultura Brasileira. Grama em tapetes - custo de produção, São Paulo, 2006. 521p.

BOWMAN, D.C. et al. Fate and transport of nitrogen applied to six warm-season turfgrasses. Crop Science, v.42, p.833841, 2002.

BERTON, R. S. et al. Peletização do lodo de esgoto e adição de $\mathrm{CaCO}$ na produção de matéria seca e absorção de $\mathrm{Zn}$, Cu e Ni pelo milho em três latossolos. Revista Brasileira de Ciência do Solo, v.21, p.685-691, 1997.

BOEIRA, R.C. Uso de lodo de esgoto como fertilizante orgânico: disponibilização de nitrogênio em solo tropical. Jaguariúna, SP. EMBRAPA. 2004. 3p. (Comunicado Técnico).

CHRISTIANS, N.E. Fundamental of turfgrass management, Chelsea, MI: Arbor Press, 1998, 301p.

CONAMA - CONSELHO NACIONAL DO MEIO AMBIENTE. Define critérios e procedimentos, para o uso agrícola de lodos de esgoto gerados em estações de tratamento de esgoto sanitário e seus produtos derivados, e dá outras providências. Brasília, DF, 2006. 41p.

DA ROS, C.O. et al. Lodo de esgoto: efeito imediato no milheto e residual na associação aveia-ervilhaca. Revista Brasileira de Ciência do Solo, v.17, p.257-261, 1993.
EMBRAPA - EMPRESA BRASILEIRA DE PESQUISA AGROPECUÁRIA - Embrapa Sistema Brasileiro de Classificação de Solos. Brasília: Embrapa Produção de Informações, 1999. 61p.

GODOY, L.J.G.; VILLAS BÔAS, R.L. Nutrição e adubação para gramados. In: SIGRA - Simpósio sobre Gramados. 2003, Botucatu, SP. Produção Implantação e Manutenção: Anais... Botucatu: Departamento de Recursos Naturais, Faculdade de Ciências Agronômicas, Universidade estadual Paulista, 2003. 1 CD-ROM.

GODOY, L.J.G. Adubação nitrogenada para produção de tapetes de grama santo agostinho e esmeralda. 2005, 106f. Tese (Doutorado em Agronomia/Agricultura) - Faculdade de Ciências Agronômicas, UNESP, Botucatu, SP.

GODOY, L.J.G. et al. Doses de nitrogênio e potássio na produção de grama esmeralda. Ciência e Agrotecnologia, v.31, n.5, p.1326-1332, 2007.

GURGEL, R.A.G. Principais espécies e variedades de grama. In: In: SIGRA - Simpósio sobre Gramados. 2003, Botucatu, SP. Produção Implantação e Manutenção: Anais... Botucatu: Departamento de Recursos Naturais, Faculdade de Ciências Agronômicas, Universidade estadual Paulista, 2003. 1 CD-ROM.

KOSKE, T.J. Sod production for Louisiana. LSU Ag Center, 1994. Acessado em 10 de dezembro de 2004. Online. Disponível em: http:/ /www.lsuagcenter.com/en/lawn_garden/commercial_horticulture/ turfgrass/sod_farming/Sod+Production+in+Louisiana.htm

MURRAY, J.J. Utilization of composted sewage sludge in sod production.. In R.W. SHEARD (ed) (INT. TURFGRASS RESEARCH CONF.), 4th, Ontario Agric. College, Proceedings... Univ: of Guelph. Ontario, 1991. p.544.

PIMENTA, C.H. Produção de gramas. In: SIGRA - Simpósio sobre Gramados. 2003, Botucatu, SP. Produção Implantação e Manutenção: Anais... Botucatu: Departamento de Recursos Naturais, Faculdade de Ciências Agronômicas, Universidade estadual Paulista, 2003. 1 CD-ROM.

SANEPAR. Companhia de Saneamento do Paraná: Manual Técnico para Utilização Agrícola do lodo de esgoto no Paraná, Curitiba, PR, 1997. 96p.

SILVA, F.C. et al. Efeito de lodo de esgoto na fertilidade de um Argissolo Vermelho-Amarelo cultivado com cana-de-açúcar. Pesquisa Agropecuária Brasileira, v.36, n.5, p.831-840, 2001.

SILVA, J. E. et al. Alternativa agronômica para o biossólido produzido no distrito federal. I - Efeito na produção de milho e na adição de metais pesados em Latossolo no cerrado. Revista Brasileira de Ciência do Solo, Viçosa, v.26, p.487-495, 2002. 